\title{
Capillary flow along rounded interior corner of right-angle under microgravity
}

\author{
Qi KANG*, Rui HOU, Li DUAN, Liang HU \\ National Microgravity Laboratory/CAS, Institute of Mechanics, Chinese Academy of Sciences, \\ Beijing 100190, China
}

\begin{abstract}
It is crucial to investigate the capillary driven flows along interior corners because of the interior corners of space fluid management devices provide the main conduits for the transfer of fluids. In many instances, the interior corners are not perfectly sharp but rather possess a degree of roundedness due to the design or fabrication. In this work, the problem of capillary flows along rounded interior corners is revisited experimentally. Four test cells which are made of PMMA are designed. The cross sections of the four cells are the same pentagon except that one $90^{\circ}$ corners are rounded with different radius $R 0$ (sharp corner), $R 2.4, R 4.8, R 6$, respectively. Four kinds of liquids are used in the microgravity drop tower tests, i.e. KF96-5 silicone oil, KF96-10, KF96-50 and Fluorinert liquid FC-70. The experimental results show that the advancing meniscus tip location of the fluid in the corner $L(\mathrm{~mm})$ is affected by container geometry and fluid properties. These experimental results are valuable to better understand the capillary flow and also can provide scientific guidance for the design and analysis of space fluid management systems.
\end{abstract}

Keywords: capillary flow, rounded interior corner, space fluid management

\section{INTRODUCTION}

A significant portion of liquid behavior in spacecraft is capillary dominated: such as liquid propellants, thermal fluids, and wastes ${ }^{[1]}$. It is therefore natural to consider designs that best exploit the spontaneous character of such capillary surfaces and flows. The 'interior corner' construct is the focus of this research because it is commonly employed in fluid system aboard spacecrafts to passively control large length scale capillary-dominated liquid in a desired manner. In most instances, the interior corners are not ideally sharp but rather possess a degree of roundedness because of the design or fabrication, as shown in Fig. 1. So far the flows in perfectly sharp interior corners have been studied extensively ${ }^{[1]-[4]}$. However, the capillary flows along rounded corners for example the impact of corner roundedness have not been fully investigated. Because the rounding of the corner has significant effect on the 'critical' contact angle ${ }^{[5]}$ and any degree of corner roundedness can prevent the spread of liquid drops in partial-wetting systems ${ }^{[6]}$, it is necessary to research the impact of roundedness on the flows along corners.

In this work, only fluids that satisfy the Concus-Finn corner-wetting condition are considered. For sharp corners the Concus-Finn condition ${ }^{[2]}$ requires that $\theta<\pi / 2-\alpha$, where $\theta$ and $\alpha$ are the contact angle and corner half-angle, respectively; this condition produces an under-pressure in the fluid that draws it spontaneously into and along the corner. But a rounded corner may prevent such a flow. Concus and Finn ${ }^{[5]}$ discussed several illustrative examples, and applied a mathematical procedure in detail to a container with a rectangular section on which the corners had been rounded; thereby they determined the effect of rounding on the 'critical' contact angle. Weislogel and Lichter ${ }^{[6]}$ found that even slight roundedness could prevent the spread of liquid drops along the corners in the case $\theta>0$. Dong and Chatzis ${ }^{[7]}$ performed both analysis and experiments studying imbibition flow in micro-scale capillary tubes of square cross sections with or without rounded corners. It is observed that the flow along rounded corners is complicated by the fact that the flow resistance varies dramatically in the flow direction. Although their experiments compare relatively well with analysis, the analytic results are strongly dependent on the numerical data of flow resistance. Recently, it is demonstrated by Chen et al. ${ }^{[8]}$ that a well-considered scaling of the cross-flow problem can lead to a local flow resistance function that is narrowly confined for all of the geometric parameters of the problem. And a similarity solution of the spontaneous

* kq@imech.ac.cn; phone: +86-10-82544112; fax: +86-10-82544096

ICEM 2008: International Conference on Experimental Mechanics 2008, edited by Xiaoyuan He, Huimin Xie, Yilan Kang, Proc. of SPIE Vol. 7375, 73751X · C 2009 SPIE · CCC code: 0277-786X/09/\$18 · doi: 10.1117/12.839078 
capillary flow in rounded corners is obtained. Furthermore, Chen et al. ${ }^{[9]}$ performed drop tower experiments to benchmark the analytical results. It is shown that the analytical predictions compare well with experiments. Hou and Duan et al. ${ }^{[10]}$ found that there were at least two regimes for the capillary flow along rounded corner, i.e. from inertial to viscous regimes.

In this paper, experimental research on the flows along rounded corners is done by a series of microgravity drop tower tests basing on the prior work ${ }^{[10]}$. The results will be presented and the impacts of the roundedness and the fluid properties will be discussed.
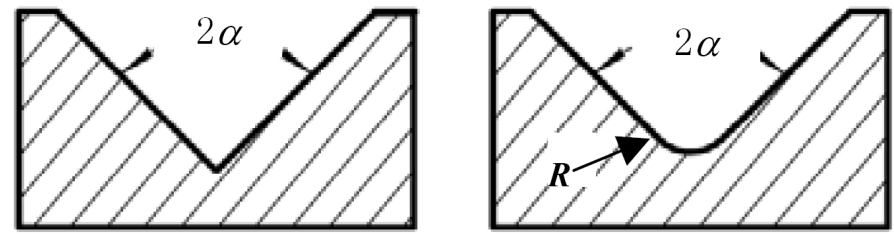

Fig. 1. Sketch of perfectly sharp corner and rounded corner
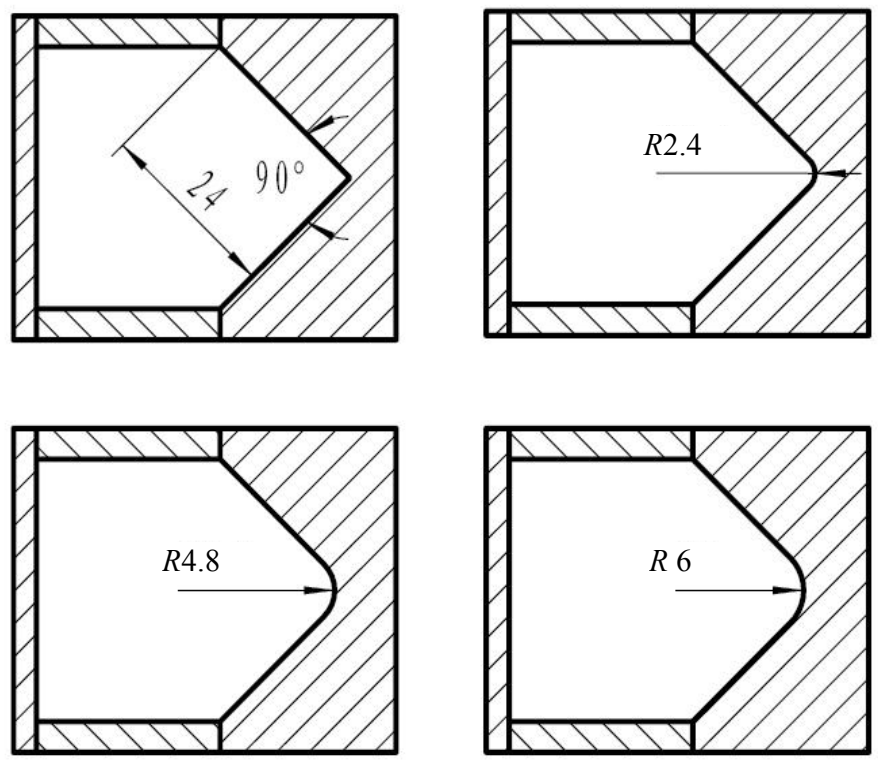

Fig. 2. Cross sections of the capillary rise test cells and dimensions (mm)

\section{CAPILLARY RISE EXPERIMENTS}

A 3.5 second drop tower facility at National Microgravity Lab of CAS to access the low-g environment for the capillary flow tests. The drop tower is approximately $124 \mathrm{~m}$ totally in height and can provide low-g environment of $10^{-5} \mathrm{~g}_{0}$.

Four transparent test cells are designed, which are made of PMMA (polymethyl methacrylate) with uniform cross section. The cross sections of the four cells are the same pentagon with interior corners of $90^{\circ}, 135^{\circ}, 90^{\circ}, 135^{\circ}$ and $90^{\circ}$ except that the middle $90^{\circ}$ corners are rounded with different corners radius $R 0$ (sharp corner), $R 2.4, R 4.8, R 6$, respectively. The size of the pentagon section is about $33.94 \mathrm{~mm} * 24 \mathrm{~mm} * 24 \mathrm{~mm} * 24 \mathrm{~mm} * 24 \mathrm{~mm}$. Fig. 2 illustrates the cross-section of the cells tested and provides some dimensions.

In order to study the impact of the liquids physical properties such as kinetic viscosity, four kinds of liquids (see table 1) are used in the tests, i.e. KF96-5 Silicone Oil, KF96-10, KF96-50 and Fluorinert liquid FC-70. 
The four test cells are installed in the drop tower apparatus. Prior to release of the apparatus into free fall, a certain amount of fluid is injected into the cells so as to fill them partly. The fluid wicks into the corners in the test cell slightly. Upon release, hydrostatic forces are essentially eliminated and capillary driven flow results. The fluid is redistributed along the corners. The flow process is backlighted by a light source and filmed with two CCD cameras (WAT-660D, Watec Co., Ltd.) at 25 frames per second. Several diffusers are used so that the light is more evenly. A schematic drawing of the setup is shown in Fig. 3. To ensure accurate meniscus tip location measurements perpendicular to the corner axis, the test cells are specially installed so that the plane bisecting the corner angle of concern is perpendicular to the camera line of sight. Quantitative data of meniscus tip location $L(\mathrm{~mm})$ are digitized directly from the video and then analyzed using image software.

Table 1. Physical properties of test liquids (25 )

\begin{tabular}{|c|c|c|c|c|c|}
\hline & $\sigma\left(\mathrm{N} / \mathrm{m} \times 10^{-3}\right)$ & $\rho\left(\mathrm{kg} \cdot \mathrm{m}^{-3}\right)$ & $\mu\left(\mathrm{kg} / \mathrm{ms} \times 10^{-3}\right)$ & $\nu\left(\mathrm{mm}^{2} / \mathrm{s}\right)$ & $\theta_{\text {stat }}$ \\
\hline $5 \mathrm{cS}$ & 19.7 & 915 & 4.575 & 5.0 & $0^{\circ}$ \\
\hline $10 \mathrm{cS}$ & 20.1 & 935 & 9.350 & 10.0 & $0^{\circ}$ \\
\hline $50 \mathrm{cS}$ & 20.8 & 960 & 47.550 & 50.0 & $0^{\circ}$ \\
\hline FC-70 & 18.0 & 1930 & 27.020 & 14.0 & $0^{\circ}$ \\
\hline
\end{tabular}

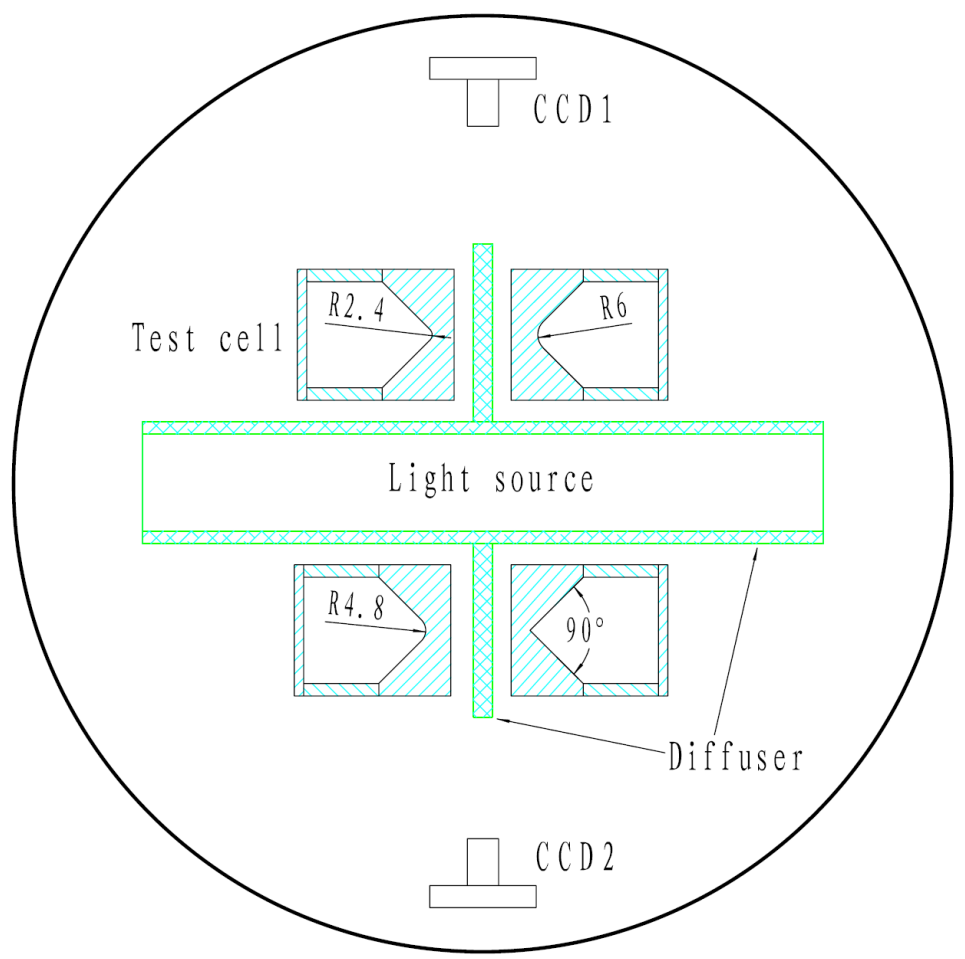

Fig. 3. Sketch of the setup of round platform for drop tower tests

\section{RESULTS AND DISCUSSIONS}

The principal measured from the film records is the advancing meniscus tip location of the fluid in the corner $L$. Some typical images taken from the film records are provided as Fig. 4 for the test of 10cS silicone oil fluid in the four test cells. From the top down, four rows of images (a), (b), (c), (d) show the results of four test cells $R 0$ (sharp corner), $R 2.4$, $R 4.8, R 6$, respectively. The left parts of Fig. 4 are the schematic drawings of the cross-sections and the field of the view 
on the right (the dashed border). The right parts of Fig. 4 are the selected frames from records for $10 \mathrm{cS}$ silicone oil at $0.6 \mathrm{~s}$ intervals.
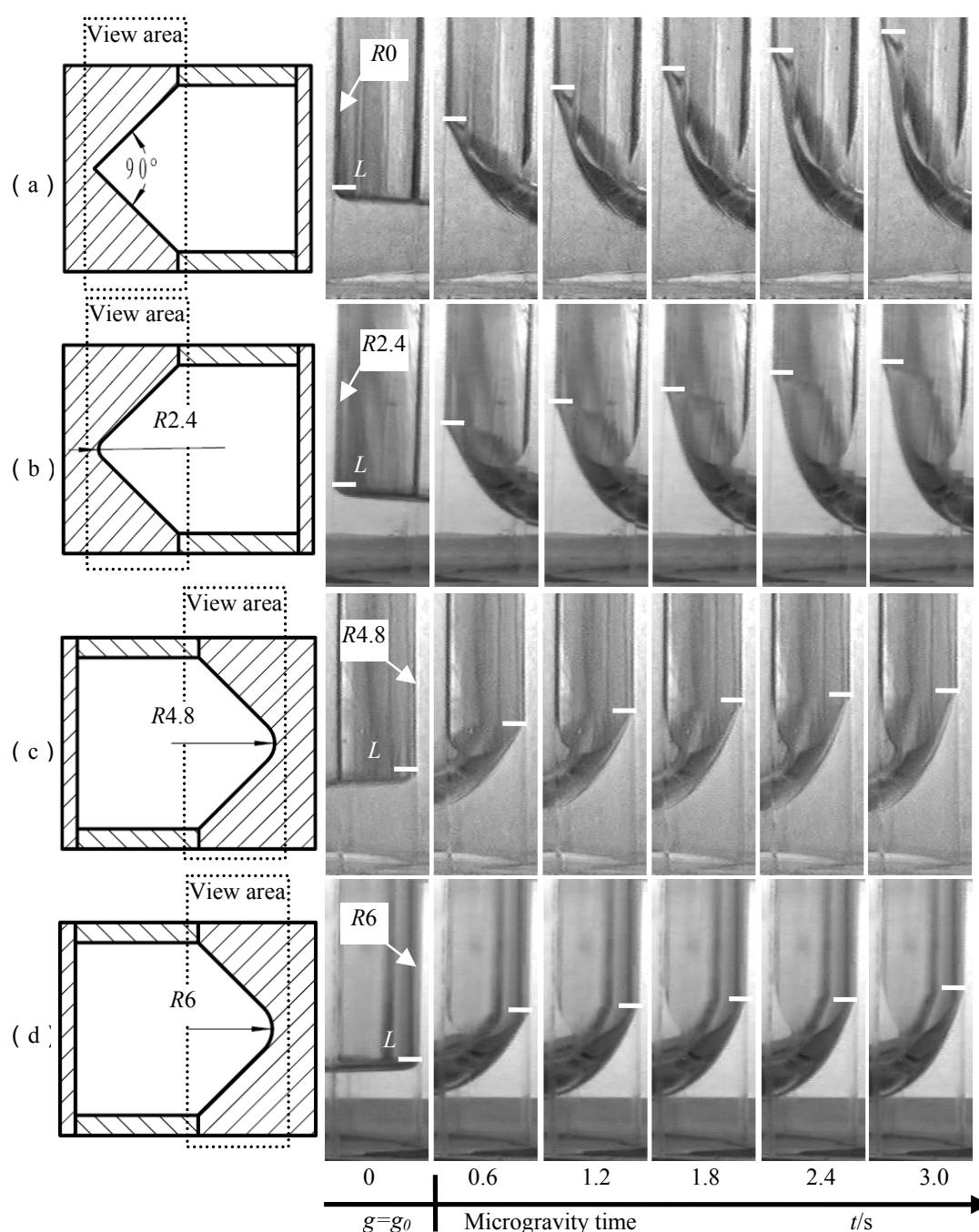

Microgravity time
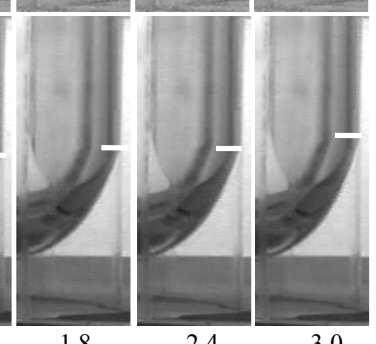

Fig. 4. From the top down, four rows of images (a), (b), (c), (d) show the results of four test cells $R 0$ (sharp corner), $R 2.4$, $R 4.8, R 6$, respectively. The left parts are the schematic drawings of the cross-sections and the field of the view on the right (the dashed border). The right parts are the selected frames from records for $10 \mathrm{cS}$ silicone oil at $0.6 \mathrm{~s}$ intervals.

Fig. 5 illustrates the impact of the corner roundedness on the tip location $L$ for tests performed when the fluid properties are fixed. It is obvious that the roundedness can prevent the climb of capillary flow along the corner. The tip velocity decreases with increasing the corner radius. The result indicates that it is necessary to conceder the importance of the corner roundedness when we design the space fluid management devices. On the other hand, the result provides another method of controlling liquid surface while doing the space fluid experiments.

Fig. 6 shows the effect of viscosity on the tip location $L$ when the test cells are the same. As anticipated, the tip velocity is greater when the fluid viscosity is smaller.

The release mechanism used in the drop tower experiments provides a nearly discontinuous reduction of gravity $\boldsymbol{g}$. So the process of capillary flow along rounded corner includes at least two regimes ${ }^{[10]}$ i.e. inertial regime and viscous regime. As far as the inertial regime is concerned, there may be two stages. At the first stage, the response of the fluid to the sudden reduction of body force is complicated and inertia plays a dominant role at the stage of the bulk meniscus reorientation. The tip location $L$ grows linearly with time $\sim t$, see Fig.5 (a), Fig. 6 (a). During the second stage, the role of 
inertia becomes weakened gradually and that of viscous becomes stronger. The tip velocity gets smaller. The relationship between $L$ and $t$ may become $\sim t^{3 / 5}$, like Weislogel ${ }^{[4]}$ said. But in this work there is no enough data to prove that. Concerns viscous regime, the role of viscous is dominant. The tip velocity becomes smaller gradually. The tip location $L$ grows with $\sim t^{1 / 2}$, see Fig. 5 (b), Fig. 6 (b). The fitting relationship is $L=c+m t^{1 / 2}$ to the data for $t>t_{\mathrm{H}}$. This experimental result compares well with the analytic prediction by Chen et al. ${ }^{[8]}$.

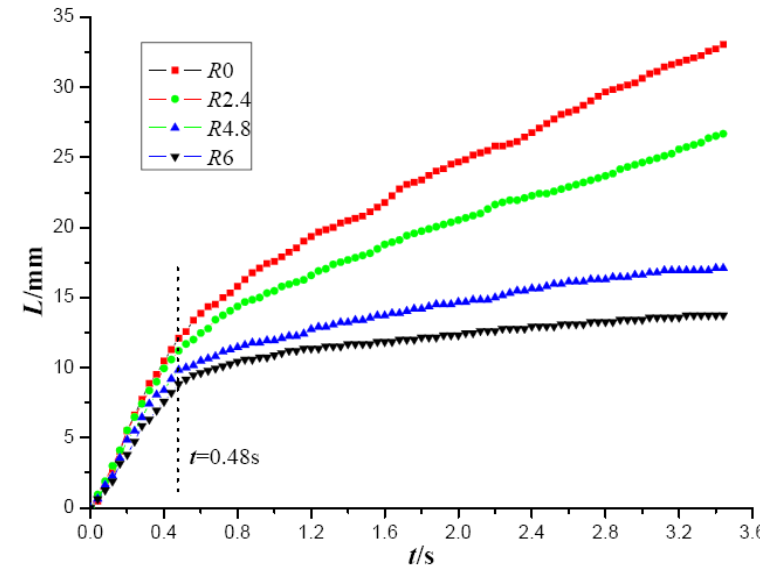

(a)

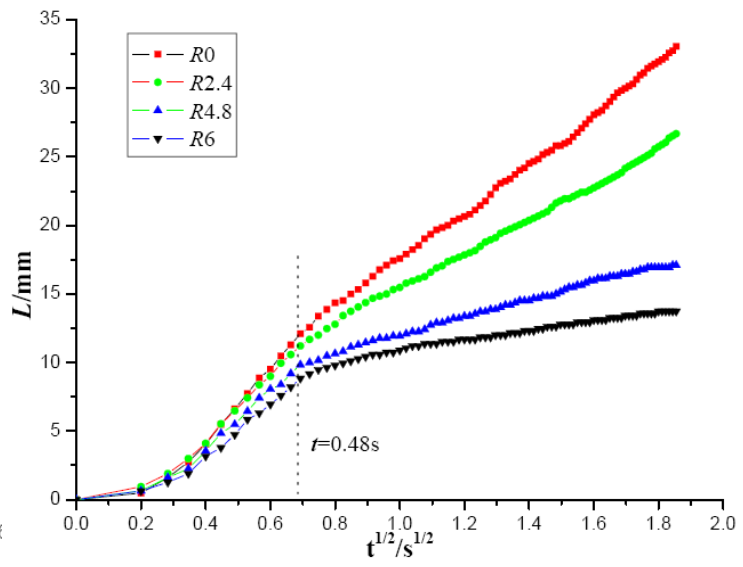

(b)

Fig. 5. The comparison of capillary test results along corners with different roundedness. (a): the tip location $L(\mathrm{~mm})$ as a function of $t(s)$. (b): the tip location $\mathrm{L}(\mathrm{mm})$ as a function of $t^{1 / 2}\left(s^{1 / 2}\right)$. Fluid: $10 \mathrm{cS}$ silicon oil.

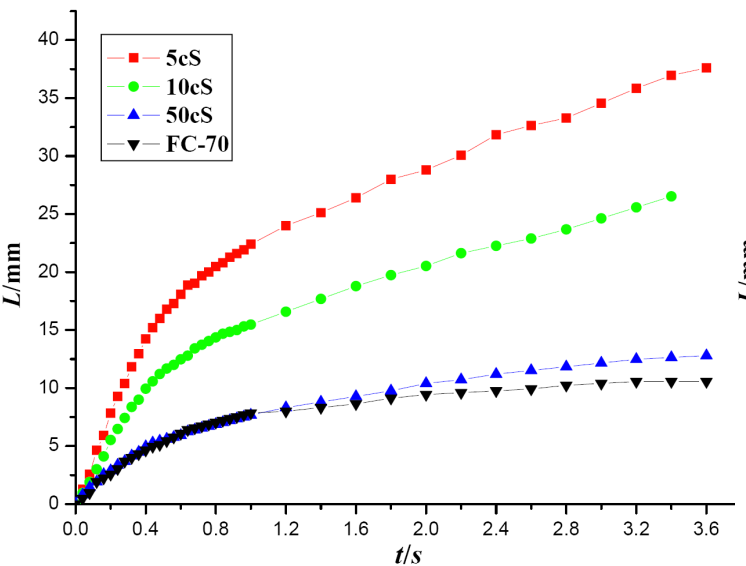

(a)

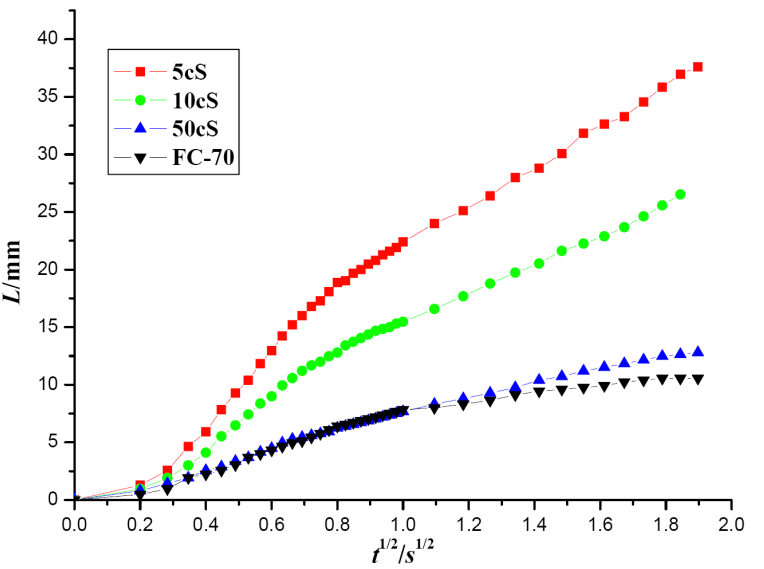

(b)

Fig. 6. Effect of viscosity on the tip location $L$ with corner roundedness is fixed. (a): the tip location $L(\mathrm{~mm})$ as a function of $t(s)$. (b): the tip location $\mathrm{L}(\mathrm{mm})$ as a function of $t^{1 / 2}\left(s^{1 / 2}\right)$. Corner radius: $R 2.4 \mathrm{~mm}$.

\section{CONCLUSIONS}

In this work, capillary flow along rounded corners is studied experimentally through several drop tower experiments. The aim of the study is to research the impacts of corner roundedness and liquid properties on the flows. Especially, the process of the capillary driven flow is discussed. With increasing the corner radius, the tip velocity decreases even to zero which is fatal to the space fluid management system design. On the other hand, this method can be used for controlling the liquid interface while doing space fluid experiments. The tip velocity is smaller when the fluid viscosity is larger. The experimental results also indicate that the process of capillary rise in rounded corner consists of at least two regimes: inertial regime $(L \sim t)$ and viscous regime $\left(L \sim t^{1 / 2}\right)$. 


\section{ACKNOWLEDGEMENTS}

The authors would like to thank the staffs of National Microgravity Lab/CAS for assistance with the drop tower experiments. This work is supported by the National Science Foundation of China $(10672171,10432060)$ and Knowledge Innovation Program of CAS (KJCX2-YW-L08).

\section{REFERENCES}

[1] Weislogel, M. M., "Capillary flow in containers of polygonal section,” AIAA J. 39(12), 2320-2326(2001).

[2] Concus, P. and Finn, R., "On the behavior of a capillary surface in a wedge," Proc. Natl. Acad. Sci. USA 63(2), $292-$ 299(1969).

[3] Concus, P. and Finn, R., "On capillary free surfaces in the absence of gravity,” Acta Math. 132, 177-198(1974).

[4] Weislogel, M. M. and Lichter, S., "Capillary flow in an interior corner," J. Fluid Mech. 373, 349-378(1998).

[5] Concus, P. and Finn, R., "Dichotomous behavior of capillary surfaces in zero gravity," Microgravity Sci. Technol. III (2), 87-92(1990).

[6] Weislogel, M. M. and Lichter. S., "A spreading drop in an interior corner: Theory and experiment." Microgravity Sci. Technol. IX (3), 175-184(1996).

[7] Dong, M. and Chatzis, I., "The imbibition and flow of a wetting liquid along the corners of a square capillary tube," J. Colloid Interface Sci. 172, 278-288(1995).

[8] Chen, Y. K., Weislogel, M. M., and Nardin, C. L., "Capillary-driven flows along rounded interior corners," J. Fluid Mech. 566, 235-271(2006).

[9] Chen, Y. K., Weislogel, M. M., and Bolleddula, D. A., "Capillary flow in cylindrical containers with rounded interior corners," $45^{\text {th }}$ AIAA Aerospace Sciences Meeting and Exhibit, Reno, Nevada, Jan. 8-11, 2007, paper AIAA2007-745(2007).

[10] Hou, R., Duan, L., Hu, L., and Kang, Q., "Capillary-driven flows along rounded interior corners in microgravity," Journal of Experiments in Fluid Mechanics, 2, 76-80(2008). 